\title{
NGF-dependent retrograde signaling: survival versus death
}

\author{
Yang Zhou ${ }^{1}$, Ting-Jia Lu' ${ }^{1}$ Zhi-Qi Xiong ${ }^{1}$ \\ ${ }^{I}$ Institute of Neuroscience and State Key Laboratory of Neuroscience, Shanghai Institutes for Biological Sciences, Chinese Academy \\ of Sciences, 320 Yueyang Road Shanghai 200031, China \\ Cell Research (2009) 19:525-526. doi: 10.1038/cr.2009.47; published online 4 May 2009
}

Nerve growth factor (NGF) was first discovered more than 5 decades ago as a molecule that promotes the survival and maturation of developing neurons in the peripheral nervous system [1]. NGF released from target cells activates tropomyosin-related kinase $\mathrm{A}$ (TrkA) on axon terminals and triggers activation of PI3K/Akt, MEK/ ERK, and PLC $\gamma$ signaling pathways. The signal then travels retrogradely along axon to cell body to promote neuronal survival [2]. However, the nature of the retrograde signal remains mysterious.

Several distinct types of retrograde signals could derive from axon terminals [3]. First, NGF itself could undergo retrograde transport from terminals to cell body thus activates intracellular TrkA receptor. Indeed, ${ }^{125}$ I-labeled NGF applied to axon terminals in vivo was found to be retrogradely transported to the neuronal cell bodies [4]. Second, NGF triggers TrkA endocytosis through binding to TrkA, and the endocytic TrkA might serve as the retrograde signal [5]. In supporting of this notion, both NGF and phosphorylated TrkA are found in endosomes [6]. Finally, NGF activates TrkA downstream signal molecules,

Correspondence: Zhi-Qi Xiong

E-mail: xiongzhiqi@ion.ac.cn which could also provide as the retrograde signals [7]. These hypotheses are not mutually exclusive, and multiple retrograde signals may exist.

In this issue, Mok and colleagues describe a fundamentally different retrograde mechanism in which NGF suppresses an apoptotic signal in distal axons [8]. Campenot's group developed compartmentalized cultures of sympathetic neurons which could segregate the distal axons from cell bodies and proximal axons by a distance of about $1 \mathrm{~mm}$. By employing this system with advantages of segregating cellular compartments, researchers could dissect the molecular mecha- nisms underlying NGF-dependent retrograde signaling. Previous studies from Campenot's laboratory demonstrated that NGF applied to distal axons of sympathetic neurons supports neuronal survival without transport of NGF towards the cell bodies or TrkA phosphorylation in the cell bodies, suggesting that NGF binding to TrkA in distal axons triggers its downstream signaling cascades locally; afterwards the signals travel retrogradely to the cell bodies and communicate with soma [9]. In the current study, the authors found that neutralizing NGF in distal segment of axons but not in the cell bodies led to activation of c-jun

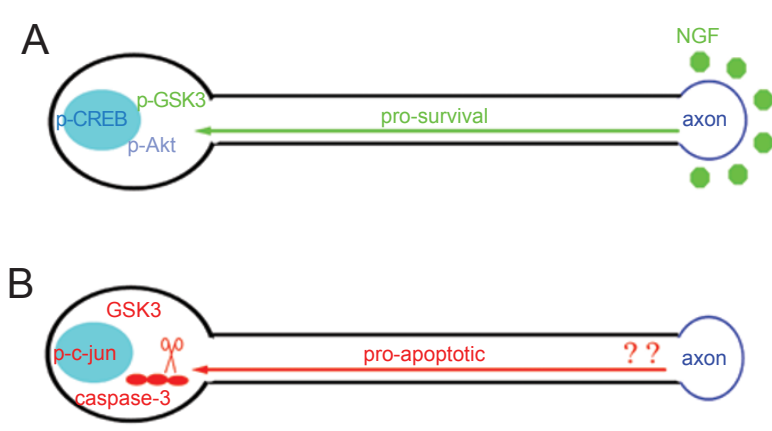

Figure 1 Schematic model of NGF-dependent sympathetic neuron survival or apoptosis. (A) In the presence of NGF in axon terminals, retrogradely transmitted pro-survival signal facilitates neuronal survival via $p$-GSK3, p-Akt and $p$ CREB signaling pathways. (B) In the absence of NGF in axon terminals, retrograde pro-apoptotic signal leads to cleavage of caspase-3, dephosphorylation of GSK3, as well as phosphorylation of c-jun, thus promoting the apoptosis of sympathetic neuron. 
and cleavage of caspase- 3 in the cell bodies as illustrated in the Figure 1 of schematic model. Interestingly, when NGF was applied to cell bodies while deprived from the distal axons at the same time, nuclear accumulation of phosphorylated c-jun was still observed, similar to cultures completely deprived of NGF. These findings are consistent with the notion that loss of NGF in the distal axons generates an apoptotic signal in the axon terminals which travels to the cell bodies and activates c-jun. However, local delivery of NGF at cell bodies protected against cell death despite the activation of c-jun, suggesting that survival signals activated by NGF in the cell bodies do not simply override the retrograde apoptotic signal but promote neuronal survival probably by acting downstream of c-jun. The axon-specific pro-apoptotic signal was blocked by treating cultures with the protein kinase $\mathrm{C}(\mathrm{PKC})$ inhibitor rottlerin, but not by down-regulating the proposed $\delta$ isoform of PKC. This inconsistency between pharmacological approach and molecular manipulation may be due to the inefficiency of siRNA knockdown or non-specific effects of pharmacological inhibitor. Further efforts to understand how rottlerin exerts its protective effect showed that NGF deprivation in axon terminals activated GSK3 in a rottlerin-sensitive manner in the distal axons. Furthermore, inhibiting GSK3 activity in distal axons or cell bodies/ proximal axons was able to prevent NGF deprivation-induced apoptosis. However, how PKC and GSK3 were activated at axon terminals were not clear.
Growing evidence suggests that NGF supports neuronal survival by activating TrkA and its downstream signals at the axon terminals which retrogradely transmit to the cell bodies. The current study demonstrates that NGF deprivation at the distal axons induces a transportable retrograde pro-apoptotic signal traveling towards the cell bodies to initiate apoptosis. Whether the inhibition of the retrograde apoptotic signal initiated at the axon terminals accounts for NGF-dependent neuronal survival or this novel mechanism operates along with other distinct pathways within the same neurons remains unknown. It is possible that sustained pro-survival signal is activated in the presence of NGF in axon terminals to antagonize endogenous pro-apoptotic signal.

Taken together, this study provides evidence in cultured sympathetic neurons that deprivation of NGF at axon terminal triggers the pro-apoptotic signal which in turn transmits to cell body. Results of this study also shed lights on neurodegenerative diseases [10]. However, several important issues remain to be solved: how does the retrograde signal transport from axon terminal to cell body? Is it in a cargo-like transport manner or a flow of continuously signaling activation along the axon segment from distal part to soma? What is the minimal amount of NGF required to antagonize the apoptotic signal? What is the molecular mechanism underlying the cross-talk between pro-survival and pro-apoptotic signals? Does neurotrophin deprivation induce retrograde pro-apoptotic signaling in sympathetic neurons in vivo? Can the same notion be applied to situations such as in nerve injury? Finding answers to these questions is of high importance, both for basic neurobiology and neurodegenerative diseases.

\section{References}

1 Levi-Montalcini R, Angeletti PU. Nerve growth factor. Physiol Rev 1968; 48:534-569.

2 Zweifel LS, Kuruvilla R, Ginty DD. Functions and mechanisms of retrograde neurotrophin signalling. Nat Rev Neurosci 2005; 6:615-625.

3 Howe CL, Mobley WC. Long-distance retrograde neurotrophic signaling. Curr Opin Neurobiol 2005; 15:40-48.

4 Campenot RB, MacInnis BL. Retrograde transport of neurotrophins: fact and function. J Neurobiol 2004; 58:217229.

5 Ginty DD, Segal RA. Retrograde neurotrophin signaling: Trk-ing along the axon. Curr Opin Neurobiol 2002; 12:268-274.

6 Ye H, Kuruvilla R, Zweifel LS, et al. Evidence in support of signaling endosome-based retrograde survival of sympathetic neurons. Neuron 2003; 39:57-68.

7 Miller FD, Kaplan DR. On Trk for retrograde signaling. Neuron 2001; 32:767-770.

8 Mok SA, Lund K, Campenot RB. A retrograde apoptotic signal originating in NGF-deprived distal axons of rat sympathetic neurons in compartmented cultures. Cell Research 2009; 19:xx$\mathrm{xx}$.

9 MacInnis BL, Campenot RB. Retrograde support of neuronal survival without retrograde transport of nerve growth factor. Science 2002; 295:1536-1539.

10 Sofroniew MV, Howe CL, Mobley WC. Nerve growth factor signaling, neuroprotection, and neural repair. Annu Rev Neurosci 2001; 24:1217-1281. 